SCIREA Journal of Physics

ISSN: 2706-8862

http://www.scirea.org/journal/Physics

May 31, 2021

Volume 6, Issue 3, June 2021

\title{
A New Understanding to the High-Pressure Hydride Superconductors - Role of Hydrogen Atoms and Effect of Pressure
}

\author{
W.Z. Shangguan ${ }^{1,2}$, Z.Cai ${ }^{1}$, Z. Q. Huang ${ }^{1}$, JiaWen Mo' ${ }^{1}$, XiangZhong Wei ${ }^{1}$ \\ ${ }^{1}$ GuangXi University of Chinese Medicine, Nanning, P.R. China \\ ${ }^{2}$ School of EEE, Nanyang Technological University, Singapore
}

\begin{abstract}
A new understanding to the origin of superconductivity in hydrogen-rich materials under high pressure is presented in this paper. Applying sufficiently high pressure to a material makes its constituent atoms get close enough so that the "close-shell inversion" effect takes place, which in turn results in superconducting state in the material. Owing to light mass and single proton in their nuclei, under high pressure the hydrogen atoms can be relatively easier squeezed into the neighborhood of heavy ones, facilitating the "close-shell inversion" effect, and in turn realizing superconductivity in the material.
\end{abstract}

Keywords: High-Pressure Superconductivity; Hydrogen-rich Material; Mechanism of Superconductivity 


\section{INTRODUCTION}

Recent landmark progress on superconductivity in hydrogen-rich materials under high pressure [1-4] was so impressive and inspiring that it has motivated intensive research on hydrides and reinvigorated the field of superconductivity since 1986, igniting the hope for room-temperature superconductivity with vast applications. Previously metallic hydrogen was predicted to be superconducting at very high temperature [5], but it was never realized because the extremely high pressure demanded to make hydrogen metallic was out of experimental capability. In 2004 Ashcroft suggested that hydrogen-rich compound containing main group elements could chemically precompress hydrogen, therefore could lower pressure required to realize superconductivity[6]. Since then hydride superconductors regained its research interests, and the breakthroughs were achieved in $\mathrm{SH}_{3}$ and $\mathrm{LaH}_{10}$ systems, with superconducting transition temperature $\left(\mathrm{T}_{\mathrm{c}}\right)$ of $203 \mathrm{~K}$ [1] and $250 \mathrm{~K}$ [2, 3], respectively, and near room temperature in the C-H-S system [4].

So far various hydrogen-rich compounds, such as $\mathrm{MgH}_{6}$ [7], $\mathrm{CaH}_{6}$ [8], $\mathrm{YH}_{6}$ [9], $\mathrm{GaH}_{3}$ [10], $\mathrm{ScH}_{9}$ [8], including the afore-mentioned $\mathrm{SH}_{3}, \mathrm{LaH}_{10}$ and organic C-H-S system as well as many others, have been studied both theoretically and experimentally. Generally the superconductivity in these hydride under high pressure is considered to be due to the phonon mechanism and understood with BCS theory, despite their major diaerences from the conventional superconductors. For example, the phonon spectrum in $\mathrm{SH}_{3}$ is both broad and has a complex structure. On the other hand, though high pressure serves as a precondition or tool to the high $T_{c}$ superconductors, which could be unstable or even do not exist under ambient pressure, its physical impact is largely limited to the understanding of squeezing the atoms and introducing density inter-crossing, giving rise therefore to some novel structures and electrical properties. However, to the best of our knowledge, there is no further understanding or discussion about the effect of high pressure.

Since Bednorz and Müller discovered high- $\mathrm{T}_{\mathrm{c}}$ cuprate in 1986 [11], the physics has entered the era of high-temperature superconductivity, while BCS theory encountered many challenges $[12,13]$, including the famous McMillan limit of $39 \mathrm{~K}$, and a variety of hightemperature superconductivity theories were proposed (see, for example, [14-16]). Discovered in 1911 by Kamerlingh Onnes [17], superconductivity remains still a puzzle and its origin is still in debate, especially in the field of so-called high-temperature superconductivity. In the so-called low-temperature superconductivity, the BCS theory was so 
successful that it has been deeply rooted among the researchers on superconductivity, despite its challenges in the high-temperature superconductivity. A fundamental and ultimate criterion, however, is that no matter high-temperature or low-temperature, all superconductors must share the same mechanism of superconductivity.

In this paper we apply the simple and universal physical model of superconductivity proposed earlier [18] to the hydrogen-rich materials under high pressure, to present a new understanding to these high-pressure hydride superconductors, and to get an insight into the role of $\mathrm{H}$ atoms and the effect of high pressure. In the next section, the physical model of superconductivity is briefly reviewed with some clarifications, and it is applied in Sec. III to the high-pressure superconductivity in hydrogen-rich materials, including other covalent systems, to show that it can be well understood within this model from the atomic scale. A concluding remark is presented in Sec. IV.

\section{ORIGIN OF SUPERCONDUCTIVITY}

From the electronic transport point of view, the grid network of lattice atoms - more precisely, the positively charged nuclei in a material — are nothing but carriers to hold the electrons, including those participating in the electrical conduction. Every individual positively charged nucleus acts just like a bridge, conveying electrons from one atomic site to another. Unlike in the normal conductor where the bridge taxes energy from electrons, in a superconductor it is completely energy-free to electrons passing by. This takes place only when the overall average inter-nuclei distance is so close that the "close-shell inversion" effect occurs. It has been shown in Ref. [18] that as long as the atoms are suc ciently close to each other, the "close-shell inversion" effect will always take place, as illustrated in Fig. 1, where the black dot in atom A represents an electron. Since the instantaneous separation distance between the lattice atom $\mathrm{A}$ and $\mathrm{B}$ is so close that this particular electron reaches a delicate equilibrium at the border of lattice atom $\mathrm{A}$ and $\mathrm{B}$, resulting in identical potential energy relative to both atoms. As a result, this electron may transfer into the orbital of atom B smoothly, moving clockwise around it (indicated by the short blue curve arrow close to atom B, while the counterpart red one indicates the possible electron transferring from atom B to atom A). Certainly this particular electron can still move around atom A anticlockwise. 


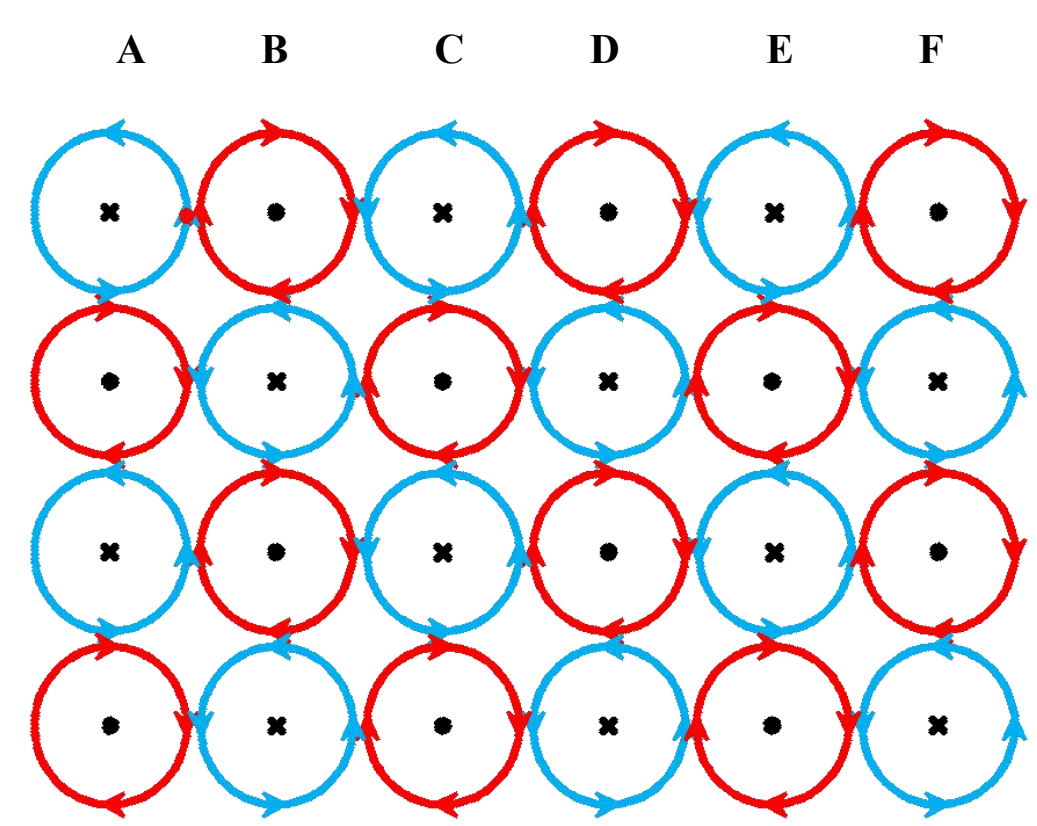

FIG. 1: Illustration of "close-shell inversion" effect. As long as inter-atomic distance is close enough, the electron either remains its original circulation direction, or transfers to the orbital of the nearest neighboring atomic site, inverting its circulation direction.

It is interesting to note that these two nearest neighboring atoms $\mathrm{A}$ and $\mathrm{B}$, or the two electrons in atomic site $\mathrm{A}$ and $\mathrm{B}$, form virtually a pair of the conduction electron, with the projections of spin and angular momentum of these two electrons in the two atomic sites opposite to each other. This somehow mimics the Cooper pair in the BCS theory. However, the core mechanism of superconductivity in our model is entirely diaerently from that of BCS theory. In our model, it is due to the "close-shell inversion" effect, while in the BCS theory, it attributes to the phonon-mediated attraction between two electrons.

Apparently, applying a high pressure to a material will cause its constituent atoms to get closer. The emergence of high-pressure hydride superconductors, which otherwise will be normal conductors, insulators or even unstable without application of su ciently high pressure, is a perfect supporter to our model of superconductivity. We conclude that a material is always superconducting as long as microscopically it contains such continuous close-enough lattice atom chains as shown in Fig. 1, no matter how zigzag they are, or even exit partially (striped or layered). So far there are two ways to bring the atoms in a material to get close enough for the "close-shell inversion" effect to take place, one is lowering the material's temperature, another is the application of a extreme high pressure. With more understanding on how atomically a large group of various atoms get relaxed under a given ambient conditions, we believe that there could possibly be alternative ways to bring the constituent atoms closer in a material. 
Physically, apart from zero resistance, another important indicator of superconductivity is Meissner effect, which means that whenever a superconductor is subject to any externally applied magnetic field (with intensity no larger than that of the critical magnetic field $\mathrm{H}_{\mathrm{c}}$ of the superconductor), its interior always remains zero magnetic field. This is self-evident for the case without any external applied magnetic field, because these 4 atomic magnetic moments, which are indicated by the central dot/cross of respective lattice atomic sites as shown in Fig. 1, are equal in magnitude with opposite directions, they cancel each other, leaving vanished magnetic field intensity macroscopically in the superconductor.

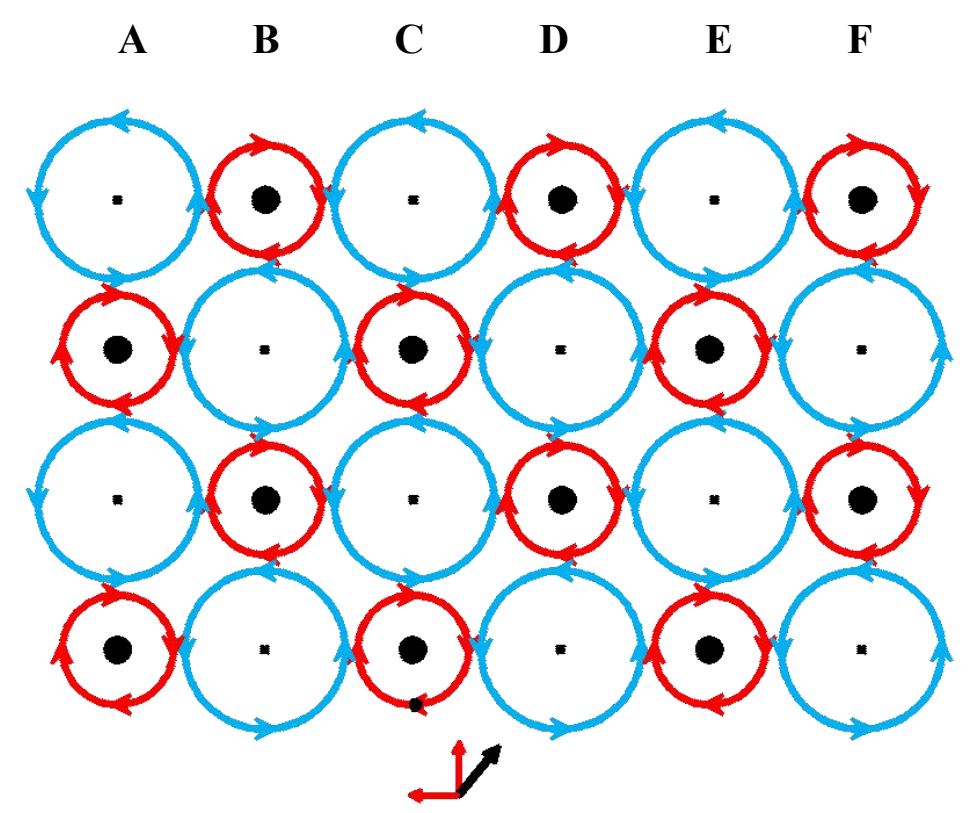

FIG. 2: Illustration of field screening mechanism. As long as a non-vanishing net magnetic field B presents, it will always induce a digerence in the magnetic moments of any two nearest neighboring atomic site, which repels the field $\mathrm{B}$.

For the case of an external magnetic field applied, these 4 magnetic moments will change accordingly, as illustrated in Fig. 2. As a result, a net repelling magnetic moment will be induced to neutralize the external field, which has been elaborated in Ref. [18]. However, here we would like to make some important clarifications. First of all the magnetic field associated with the conduction electrons around every nuclei is treated as individual magnetic moments and are denoted in the center of respective atomic site, regardless of that microscopically there might be some fluctuations spatially. Therefore, in the equilibrium case, i.e., no external applied field, it is no doubt that macroscopically the interior field vanishes. As in the case of external field applied which is shown in Fig. 2, although the magnetic moments of, for example, A and B are located at diderent positions microscopically, taking into account that 
the dimensions of atom A or B is very small, the induced net repelling magnetic field may be approximated

$$
\Delta B=B_{A}-B_{B}=\frac{1}{2} \mu_{0} e v\left(\frac{1}{R_{A}^{2}}-\frac{1}{R_{B}^{2}}\right) \quad \Delta B
$$

with effective electron circulation radius $R_{A}$ and $R_{B}$ for atom $A$ and $B$, respectively. As long as the net magnetic field is non-zero, it will always give rise to a diaerence in $R_{A}$ and $R_{B}$, which according to Eq. (1) will always result in a repelling magnetic field $\Delta \mathrm{B}$. In this way, the externally applied magnetic is gradually neutralized from the surface of the superconductor to its interior, where the external field is fully screened.

\section{EFFECT OF PRESSURE AND ROLE OF HYDROGEN}

It is interesting to note that so far all systems with $\mathrm{T}_{\mathrm{c}}>200 \mathrm{~K}$ under pressure are hydrogenrich compounds, typically the covalent $\mathrm{H}_{3} \mathrm{~S}$ and ionic clathrate $\mathrm{LaH}_{10}$. Some other classes of material can also give high $\mathrm{T}_{\mathrm{c}}$, one is the covalent metals, such as $\mathrm{MgB}_{2}$, which is driven by strongly covalent-bonding/antibonding states crossing the Fermi energy [19]. Besides, covalent hydrogen-rich organic-derived materials are another class of high- $\mathrm{T}_{\mathrm{c}}$ materials that combine the advantages of covalent metals and metal superhydrides [20, 21]. For example, the photochemically synthesized $\mathrm{C}-\mathrm{S}-\mathrm{H}$ system becomes superconducting with its highest critical temperature around $290 \mathrm{~K}$ at about $270 \mathrm{GPa}$ [4]. A common feature of all these classes of materials is the existence of covalent bonds.

On the other hand, from the physical model of superconductivity presented in Sec. II, one can find that it involves readily the features of covalent bond, because the conduction electron is shared by the two nearest-neighboring lattice atoms, i.e., the electron either continues to move around atom $\mathrm{A}$ in the original circulation direction, or transfers to its nearest-neighboring atom $\mathrm{B}$ and moves around it in the opposite circulation direction, in a way what we call "close-shell inversion".

Ashcroft' suggestion of using metallic hydrogen was based on the isotope effect with high phonon frequency, which was derived from the BCS theory, where electron-electron paring via electron-phonon coupling is responsible for the origin of superconductivity. However, we argue here that, owing to light mass and single proton in their nuclei, the hydrogen atoms can be relatively easily squeezed into the neighborhood of heavy ones such as sulfur or lanthanum atoms, forming a so-called cage of hydrogen atoms around them, and in turn passages for the 
conduction electrons to take the "close-shell inversion" effect, realizing thereafter superconductivity in the material. Being the lightest one among all elements, the hydrogen nucleus possesses the least attraction to other electrons except for its own single one. Therefore, hydrogen is the easiest candidate among all elements.

Study shows that the $\mathrm{H}$ atoms form sodalitelike clathrate cages consisting of weakly covalent $\mathrm{H}-\mathrm{H}$ bonds with a $\mathrm{H}-\mathrm{H}$ distance about $1.2 \AA$ [8], while some other calculations indicate that the bond length between $\mathrm{H}-\mathrm{H}$ is about 0.78 to $1.27 \AA$ under $300 \mathrm{GPa}$ [22- 24]. These results suggest that the $\mathrm{H}$ atoms are very close to each other relative to their own dimensions. As a result, these $\mathrm{H}$ atoms, which combine the heavy ones such as sulfur or lanthanum atoms, are actually sufficiently close to each other so that the "close-shell inversion" effect occurs easily for the conduction electrons. The reason why hydrogen-rich materials stood out recently to yield high $\mathrm{T}_{\mathrm{c}}$ superconductors under high pressure hence becomes manifesting.

The variation of microscopic distances among various kinds of atoms in a material by imposing an extreme high pressure comparable to that in the earth core, as has been simulated theoretically [22-24] and demonstrated experimentally [1-4], will undoubtedly change the constituent inter-atomic distances, and in turn the stoichiometry of the material being studied, and thus alter its chemical and physical properties. On the other hand, as has been mentioned in Sec. II, as long as it contains microscopically continuous close-enough lattice atomic chains in a material as shown in Fig. 1, no matter how zigzag they are, or even exist partially (striped or layered), the conduction electrons will take naturally the "close-shell inversion" effect, and this material will be superconducting. The application of an extremely high pressure over the material is just to manage to make the atoms in the material getting su $\varnothing$ ciently close to each other, forming a path for the conduction electrons to take naturally the "close-shell inversion" effect. From this point of view, it is quite straightforward to anticipate that in principle most material, such as some "exotic" ones like plastic, rubber, stone or ceramic, will become superconducting under su $\varnothing$ ciently high pressure even at room temperature. We also expect that in the near future many additional binary materials will be synthesized by sandwiching light nuclei other than hydrogen into heavy ones, but it may demand even higher pressure.

Since microscopically all materials are consisted of one kind or various kinds of atoms, some interesting questions may raise reasonably: under a given ambient conditions, say, a particular temperature and pressure, what determines the typical distances between these various kinds of atoms? For example, at the room temperature ambient conditions why hydrogen atoms 
gather in gaseous state macroscopically and the distance between these atoms is relatively so big, while copper atoms in solid state and the distance between them is comparably much smaller. This is not a trivial question which seems to have been solved by the Schrodinger equation, or by many-body quantum theory. Put alternatively, if a group of various kinds of atoms are put together and a macroscopic stable state is reached, what microscopically determines the distances of these atoms and hence macroscopically the physical and chemical properties of the material composed of this group of atoms? And what happens to the electrons in these atoms? These questions might be too complicated for us to answer at the moment, but hopefully will become more reachable in the future.

We believe that the nature has simple rules for the system containing huge number of various kinds of atoms to settle down. The present computational methods to these systems by the many-body theory is undoubtedly so complex and impracticable that entails much simplification and approximation, such as density functional theory method and many other quantum chemistry calculation approaches. A new framework of theory is expected to tackle the many particle systems.

\section{CONCLUSIONS}

To summarize, we have presented a new understanding to the origin of superconductivity in hydrogen-rich materials under high pressure. We argue that applying a sufficiently high pressure to a hydride material will bring the constituent atoms close enough, though may result in chemically stoichiometry change, so that the "close-shell inversion" effect will take place, which in turn results in superconducting state in the material. Due to light mass and single proton in their nuclei, under high pressure the hydrogen atoms can be relatively easily squeezed into the neighborhood of heavy ones such as sulfur or lanthanum atoms, forming a so-called cage of hydrogen atoms around them, and in turn passages for the conduction electrons to take the "close-shell inversion" effect, realizing thereafter superconductivity in the material. Hydrogen is thus the perfect candidate among all elements.

We conclude that the origin responsible for superconductivity in a material is not due to the electron-paring mechanism via electron-phonon coupling. Instead, it attributes to what we call "close-shell inversion". Superconductivity requires no electron pairs, but does the inversion of conduction electrons between two nearest-neighboring atomic sites. However, to realize a higher, or even the long-sought room-temperature superconductivity at ambient pressure 
conditions, more understanding is needed on what determine the inter-lattice atomic distance in a material.

\section{Acknowledgments}

WZS is grateful to W.Z. Jiang for his research suggestion on this topic.

\section{References}

[1] Drozdov, A. P.; Eremets, M. I.; Troyan, I. A.; Ksenofontov, V.; Shylin, S. I. Sep 2015, 525(7567), 73-76.

[2] Drozdov, A. P.; Kong, P. P.; Minkov, V. S.; Besedin, S. P.; Kuzovnikov, M. A.; Mozaøari, S.; Balicas, L.; Balakirev, F. F.; Graf, D. E.; Prakapenka, V. B.; Greenberg, E.; Knyazev, D. A.; Tkacz, M.; Eremets, M. I. May 2019, 569(7757), 528-531.

[3] Somayazulu, M.; Ahart, M.; Mishra, A. K.; Geballe, Z. M.; Baldini, M.; Meng, Y.; Struzhkin, V. V.; Hemley, R. J. Jan 2019, 122, 027001.

[4] Snider, E.; Dasenbrock-Gammon, N.; McBride, R.; Debessai, M.; Vindana, H.; Vencatasamy, K.; Lawler, K. V.; Salamat, A.; Dias, R. P. Oct 2020, 586(7829), 373-377.

[5] Ashcroft, N. W. Dec 1968, 21, 1748-1749.

[6] Ashcroft, N. W. May 2004, 92, 187002.

[7] Feng, X.; Zhang, J.; Gao, G.; Liu, H.; Wang, H. RSC Adv. 2015, 5, 59292-59296.

[8] Wang, H.; Tse, J. S.; Tanaka, K.; Iitaka, T.; Ma, Y. Proceedings of the National Academy of Sciences 2012, 109(17), 6463-6466.

[9] Li, Y.; Hao, J.; Liu, H.; Tse, J. S.; Wang, Y.; Ma, Y. May 2015, 5(1), 9948.

[10] Szczec'sniak, R.; Durajski, A. P. nov 2013, 27(1), 015003.

[11] Bednorz, J.; Müller, K. jun 1986, 64, 189-193.

[12] Anderson, P. W. Science 2007, 316(5832), 1705-1707.

[13] Hirsch, J. E. apr 2020, 130(1), 17006.

[14] Anderson, P. W.; Baskaran, G.; Zou, Z.; Hsu, T. Jun 1987, 58, 2790-2793.

[15] Anderson, P. W. Science 1987, 235(4793), 1196-1198.

[16] Anderson, P. W.; Abrahams, E. Jun 1987, 327, 363.

[17] Onnes, H. K. Commun. Phys. Lab. Univ. Leiden 1911, 12, 1.

[18] Shangguan, W. Z. Modern Physics Letters B 2019, 33(32), 1950393. 
[19] Nagamatsu, J.; Nakagawa, N.; Muranaka, T.; Zenitani, Y.; Akimitsu, J. Mar 2001, 410(6824), 63-64.

[20] Cui, W.; Bi, T.; Shi, J.; Li, Y.; Liu, H.; Zurek, E.; Hemley, R. J. Apr 2020, 101, 134504.

[21] Sun, Y.; Tian, Y.; Jiang, B.; Li, X.; Li, H.; Iitaka, T.; Zhong, X.; Xie, Y. May 2020, 101, 174102.

[22] Duan, D.; Liu, Y.; Tian, F.; Li, D.; Huang, X.; Zhao, Z.; Yu, H.; Liu, B.; Tian, W.; Cui, T. Nov 2014, 4(1), 6968.

[23] Li, Y.; Hao, J.; Liu, H.; Li, Y.; Ma, Y. The Journal of Chemical Physics 2014, 140(17), 174712 .

[24] Muramatsu, T.; Wanene, W. K.; Somayazulu, M.; Vinitsky, E.; Chandra, D.; Strobel, T. A.; Struzhkin, V. V.; Hemley, R. J. The Journal of Physical Chemistry C 2015, 119(32), $18007-18013$. 